\title{
Mittendorf's dot and posterior embryotoxon - incidental findings in a patient with a potentially occludable anterior chamber angle
}

\author{
Maciej Czepita, Anna Szewczyk, Joanna Twarużek \\ $2^{\text {nd }}$ Department of Ophthalmology, Pomeranian Medical University, Szczecin, Poland
}

\begin{abstract}
Mittendorf's dot and posterior embryotoxon are both congenital abnormalities affecting the anterior segment, which arise during early foetal life. We report of a 36-year-old female patient newly diagnosed as having a potentially occludable anterior chamber angle in the right eye and coexisting Mittendorf's dot and posterior embryotoxon. The patient elected to undergo prophylactic laser iridotomy in the affected eye. These findings may serve as additional factors to consider in the management of patients with narrow anterior chamber angles.
\end{abstract}

KEY WORDS: Mittendorf's dot, posterior embryotoxon, angle-closure

Ophthalmol J 2017; Vol. 2, No. 4, 110-112

\section{INTRODUCTION}

Mittendorf's dot is a benign congenital lesion usually presenting as a small white or translucent opacity at the posterior lens capsule just nasal to the central visual axis [1]. It is a clinical sign of a mild form of persistent foetal vasculature syndrome (PFV) [2] and does not cause visual disturbances.

Posterior embryotoxon is a central thickening and displacement of Schwalbe's line [3]. It is visible during slit-lamp examination as a concentric line anterior to the limbus of the cornea. It can be associated with Axenfeld-Rieger syndrome [4] or Alagille syndrome [5]. It may also be an isolated finding in normal patients. It does not interfere with vision.

A potentially occludable anterior chamber angle is a narrow angle judged to be particularly at risk of closure based on gonioscopic examination [6]. It is a condition warranting special attention. Patients with this condition can be managed by observation alone or by performing a laser iridotomy.

Here we describe a case of Mittendorf's dot and posterior embryotoxon in a patient newly diagnosed as having a potentially occludable anterior chamber angle.

\section{CASE REPORT}

A 36-year-old woman with a family history of glaucoma presented with complaints of mild episodic pain in her right eye lasting for over one month. Her uncorrected distance visual acuity (UDVA) in her right eye was 20/20 and 20/20 in her left eye. Intraocular pressure in her right eye was $21 \mathrm{mmHg}$ and $17 \mathrm{mmHg}$ in her left eye.

Slit-lamp biomicroscopy examination revealed a posterior embryotoxon in her right eye as well as a Mittendorf's dot on the nasal side of the posterior lens capsule after pupillary dilatation with $1 \%$ tropicamide and 10\% phenylephrine (Fig. 1). On gonioscopic the patient was found to have a narrow angle in all four quadrants in the right eye - B20s pigment $2+$ according to Spaeth classification and a slightly wider angle - C30s pigment $2+$ in her left eye according to Spaeth classification (Fig. 2). 
A

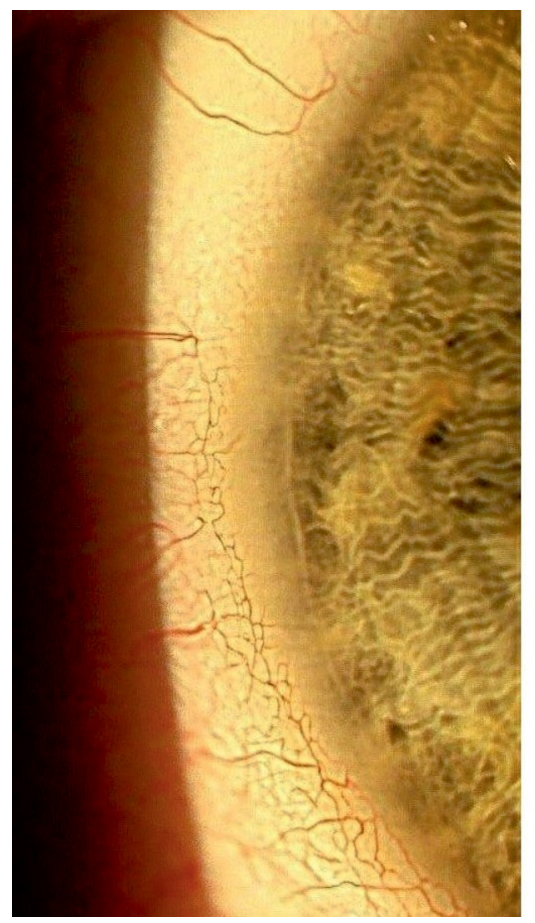

B

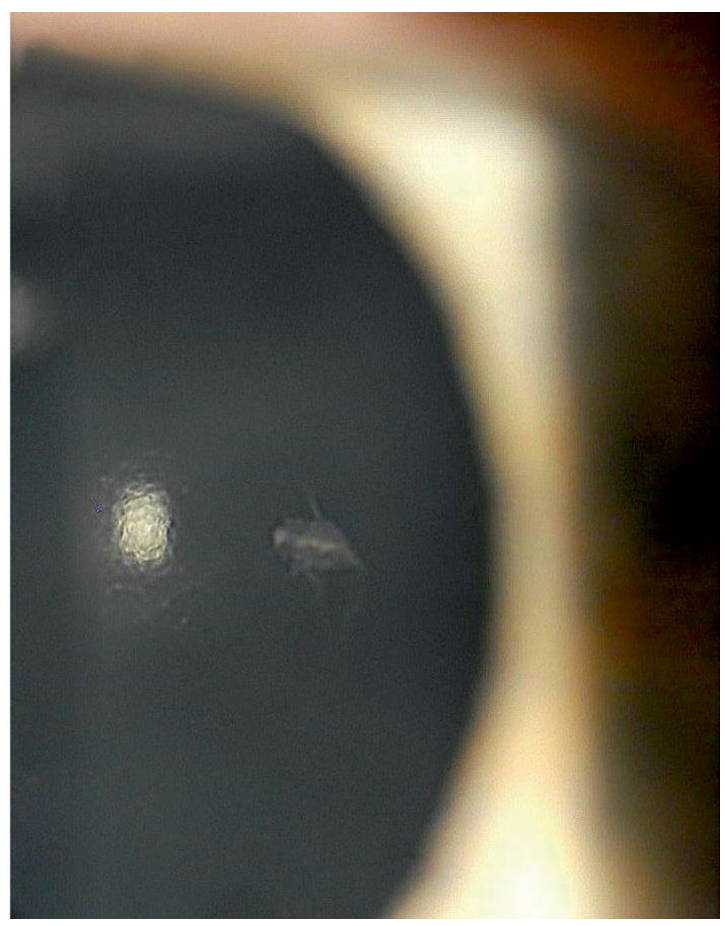

FIGURE 1. Slit-lamp image of the right eye demonstrating a posterior embryotoxon in image A and a Mittendorf's dot in image B

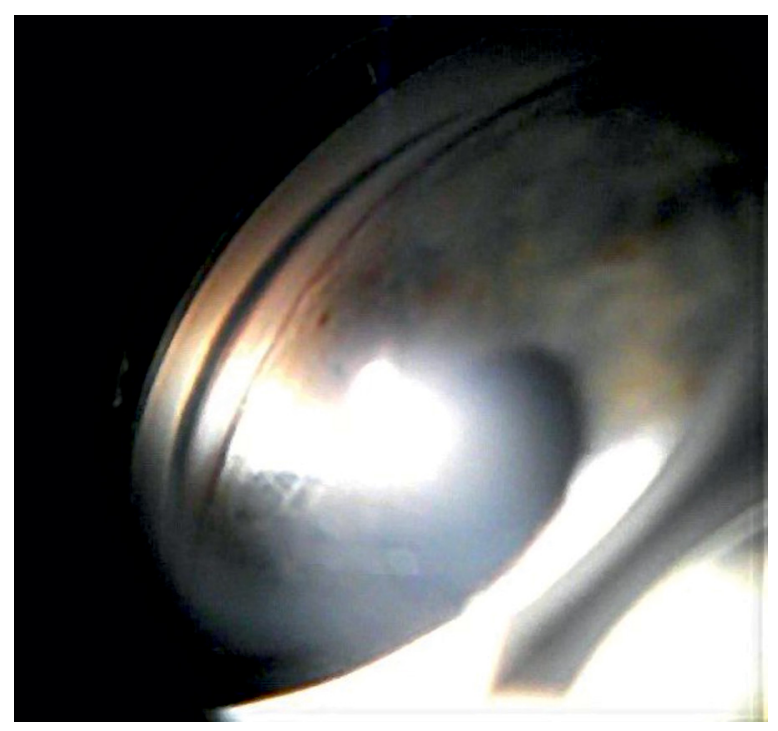

FIGURE 2. Gonioscopic view of the right eye displaying a narrow anterior chamber angle

This was later also confirmed on anterior segment optical coherence tomography (AS-OCT) examination, as seen in Figure 3. Slit-lamp examination of her left eye was unremarkable.

Based on the gonioscopic findings and results of the AS-OCT examination we diagnosed the patient as having a potentially occludable anterior cham- ber angle in the right eye. The patient elected to have a prophylactic argon laser peripheral iridotomy (ALPI) performed in her right eye. We continue to closely monitor this patient.

\section{DISCUSSION}

A Mittendorf's dot is a remnant of the hyaloid artery system, which arises from the primary vitreous during foetal life [7]. Once the secondary vitreous fills the vitreous cavity, the primary vitreous along with the hyaloid artery system get pushed anteriorly and disappear usually around the fourth month of gestation. Sometimes, what remains after this process is seen as a Mittendorf's dot.

The posterior embryotoxon consists histologically of a central collagen core surrounded by a thin Descemet's layer and is separated from the anterior chamber by a layer of endothelium [8]. Both Descemet's membrane and corneal endothelium arise from the paraxial mesenchyme. The formation of the anterior chamber angle begins between month three and four of gestation [9]. It is a lengthy process during which the mesenchyme migrates between the surface ectoderm and crystalline lens. During this time a maldevelopment such as a posterior embryotoxon may arise. 


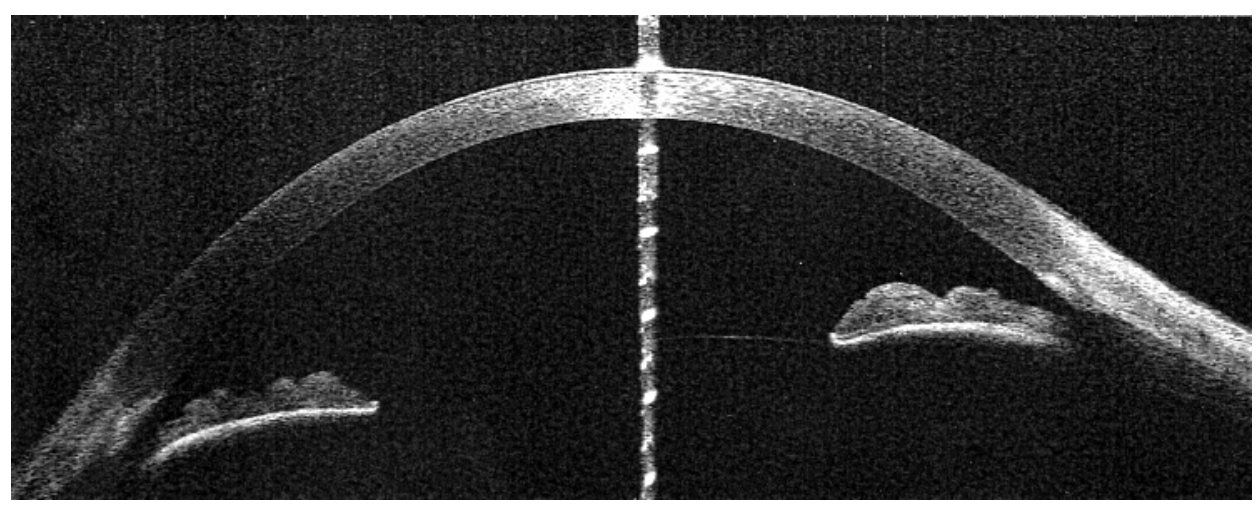

FIGURE 3. A potentially occludable anterior chamber angle of 20 degrees. Anterior segment optical coherence tomography image of the right eye

Both Mittendorf's dot and posterior embryotoxon are usually incidental findings on slit-lamp examination. These lesions do not interfere with vision and are mostly isolated and sporadically occur in healthy individuals. However, these findings should not be overlooked because they can signal other congenital anterior chamber abnormalities. Therefore, detection of these lesions should warrant a detailed examination including gonioscopy and ophthalmoscopy. The presence of these findings may be an additional factor to take into account in the management of patients with narrow anterior chamber angles.

\section{LIST OF ABBREVIATIONS}

PFV - Persistent foetal vasculature

UDVA - Uncorrected distance visual acuity

AS-OCT - Anterior segment optical coherence tomography

ALPI - Argon laser peripheral iridotomy

\section{REFERENCES}

1. Wright KW, Spiegel PH. Pediatric Ophthalmology and Strabismus. 2nd ed. Springer, New York 2003: 456.

2. Singh $S$, Hasanreisoglu M, Pointdujour $R$, et al. Stunning Visualization of Fetal Hyaloid Artery. Retina Today. 2015: 44-46.

3. Burian $H M$, Braley AE, Allen L, et al. External and gonioscopic visibility of the ring of Schwalbe and the trabecular zone; an interpretation of the posterior corneal embryotoxon and the so-called congenital hyaline membranes on the posterior corneal surface. Trans Am Ophthalmol Soc. 1954; 52(6): 389-428, indexed in Pubmed: 13274432.

4. Song $W$, Hu X. The rare Axenfeld-Rieger syndrome with systemic anomalies. Medicine. 2017; 96(33): e7791, doi: 10.1097/ md.0000000000007791.

5. Ho DK, Levin AV, Anninger WV, et al. Anterior Chamber Pathology in Alagille Syndrome. Ocul Oncol Pathol. 2016; 2(4): 270-275, doi: 10.1159/000446804, indexed in Pubmed: 27843908.

6. Carlesimo SC, Di Santo L, Bruni P, et al. Nd: Yag laser iridotomy in Shaffer-Etienne grade 1 and 2: angle widening in our case studies. Int J Ophthalmol. 2015; 8(4): 709-713, doi: 10.3980/j.issn.22223959.2015.04.12, indexed in Pubmed: 26309867.

7. Khurana AK. Comprehensive Ophthalmology. 4th. ed. New Dehli: New Age International (P) Ltd. : 9.

8. Rennie CA, Chowdhury S, Khan J, et al. The prevalence and associated features of posterior embryotoxon in the general ophthalmic clinic. Eye (Lond). 2005; 19(4): 396-399, doi: 10.1038/sj.eye.6701508, indexed in Pubmed: 15309023.

9. Weinreb RN, Friedman DS. Angle Closure and Angle closure Glaucoma. Kugler Publications, The Hague 2006: 65-69. 